\title{
ADAPTIVE KALMAN SMOOTHING OF AR SIGNALS DISTURBED BY IMPULSES AND COLORED NOISE
}

\author{
G. Doblinger \\ Institut für Nachrichtentechnik und Hochfrequenztechnik \\ Vienna University of Technology \\ Gusshausstr. 25, E389, A-1040 Vienna, Austria \\ gerhard.doblinger@tuwien.ac.at
}

\begin{abstract}
In this paper, we describe a new and computationally efficient adaptive system for the enhancement of autoregressive (AR) signals which are disturbed by additive white or colored noise and impulsive noise. The system is comprised of an adaptive Kalman filter operating as a fixed lag smoother and a subsystem for AR parameter estimation. A superior performance is achieved by implementing a feedback loop between the Kalman filter output and the parameter estimation. Accordingly, the AR parameters are obtained from the enhanced signal and the influence of the disturbing noise on the parameter estimation is damped down. Impulsive noise is suppressed by an outlier detection scheme and by freezing the Kalman filter update during presence of impulses. The subsystem for AR parameter estimation can operate in a block processing mode or on a sample per sample basis. Another advantage of the adaptive Kalman filter is its tracking capability for short-time stationary signals.
\end{abstract}

\section{INTRODUCTION}

Many natural signals like speech, music, and seismic signals can be sufficiently represented by autoregressive (AR) models. In many practical situations, however, these signals are corrupted by additive noise and must be enhanced for further processing.

In this paper, we describe such an enhancement algorithm which is based on an adaptive Kalman filter (fixed lag smoother). Because of its high flexibility, the Kalman filter is very well suited for signal enhancement purposes. The key point for the performance of such a signal enhancement system is the estimation of the AR parameters in the presence of noise. Since the AR model is built into the structure of the Kalman filter, we must ensure that the estimated AR parameters yield stable filters.

The novelty of our approach is the proper combination of a Kalman filter and a parameter estimation procedure which allows for tracking of nonstationary signals. Furthermore, our system is designed to suppress both white or colored noise and impulsive noise. The inclusion of colored noise and impulsive noise is an extension to the system we presented in [8]. Our system does not require an extended Kalman filter as used in [7] or an iterated parameter estimation procedure [6]. It is computationally efficient and may be implemented for real-time operation in the audio frequency range using integrated digital signal processors.

We first present a computationally efficient set of Kalman fixed-lag smoother equations fitted for use with AR signal and noise models. Next, we modify the Kalman fixed-lag smoother by including an impulsive noise suppression scheme based on a method proposed in [7]. Afterwards, the block diagram of the entire signal enhancement system is discussed in some detail. Finally, we present typical experimental results to illustrate the performance of the adaptive Kalman filter.

\section{KALMAN FIXED-LAG SMOOTHER FOR AR SIGNAL AND NOISE MODELS}

In this section, we characterize both the desired signal and the disturbing noise by AR signal models. These models are then used to devise a Kalman filter for optimal suppression of the disturbing noise. At the moment we assume that all model parameters are known. Later on we will show how these parameters can be estimated from the noisy signal.

Given the noisy signal by

$$
y(n)=s(n)+v(n)
$$

we represent the desired signal $s(n)$ by a $p^{t h}$ order AR process

$$
\begin{aligned}
s(n+1) & =\sum_{k=1}^{p} a_{k}(n) s(n-k+1)+u(n) \\
& =\mathbf{a}^{T}(n) \mathbf{s}(n)+u(n)
\end{aligned}
$$

where $\mathbf{a}(n)=\left(a_{1}(n), \ldots, a_{p}(n)\right)^{T}$ denotes the time dependent AR parameter vector and the signal data vector is given by $\mathbf{s}(n)=(s(n), \ldots, s(n-p+1))^{T}$. Similarly, the additive colored Gaussian noise $v(n)$ is modeled as a $q^{t h}$ order AR 
process

$$
\begin{aligned}
v(n+1) & =\sum_{k=1}^{q} b_{k}(n) v(n-k+1)+w(n) \\
& =\mathbf{b}^{T}(n) \mathbf{v}(n)+w(n) .
\end{aligned}
$$

Normally, AR model orders are not known exactly, and selecting $p=4 \ldots 20$ and $q=1 \ldots 4$ may be suitable in most practical situations. The driving noise sequences $u(n), w(n)$ of the AR models are assumed to be zero mean white Gaussian noise processes with variances $\sigma_{u}^{2}(n)$, and $\sigma_{w}^{2}(n)$, respectively. We further assume that only the disturbing noise model parameters $\sigma_{w}^{2}(n)$ and $\mathbf{b}(n)$ must be known in advance, i.e. they are estimated during absence of the desired signal $s(n)$. All other parameters are supplied by our adaptive algorithm. We prefer to use a Kalman fixed-lag smoother instead of the Kalman filter, since smoothing involves additional data in the estimation procedure. As a consequence, the estimation error variance is further decreased, and with a large lag length (delay) the performance approaches that of a noncausal Wiener filter for stationary signals [3].

In order to obtain a Kalman fixed-lag smoother we represent the AR signal model (2) in state-space form

$$
\mathbf{s}(n+1)=\mathbf{A}(n) \mathbf{s}(n)+(u(n), 0, \ldots, 0)^{T} .
$$

In contrast to (2), the $d+1$ dimensional state vector $\mathbf{s}(n)$ now consists of the current signal sample $s(n)$ and $d$ delayed samples where $d \geq p$ is the smoother delay. Comparison of the state-space model (4) with the scalar signal model (2) immediately unveils the following structure of the $(d+1) \times(d+1)$ signal state matrix $\mathbf{A}(n)$ :

$$
\mathbf{A}(n)=\left(\begin{array}{ccccccc}
a_{1}(n) & \cdots & a_{p}(n) & 0 & \cdots & 0 & 0 \\
1 & \cdots & 0 & 0 & \cdots & 0 & 0 \\
\vdots & \ddots & & & & & \vdots \\
0 & & 1 & 0 & \cdots & 0 & 0 \\
0 & & 0 & 1 & \cdots & 0 & 0 \\
\vdots & & & & \ddots & & \vdots \\
0 & \cdots & 0 & 0 & \cdots & 1 & 0
\end{array}\right)
$$

Likewise, the noise model (3) can be written in state-space form

$$
\mathbf{v}(n+1)=\mathbf{B}(n) \mathbf{v}(n)+(w(n), 0, \ldots, 0)^{T}
$$

with the $q \times q$ noise state matrix

$$
\mathbf{B}(n)=\left(\begin{array}{cccc}
b_{1}(n) & \cdots & b_{q-1}(n) & b_{q}(n) \\
1 & \cdots & 0 & 0 \\
\vdots & \ddots & 0 & \vdots \\
0 & \cdots & 1 & 0
\end{array}\right)
$$

Introducing the augmented state vector $\mathbf{x}(n)$ and driving noise vector $\mathbf{w}(n)$

$$
\mathbf{x}(n)=\left(\begin{array}{c}
\mathbf{s}(n) \\
\mathbf{v}(n)
\end{array}\right), \quad \mathbf{w}(n)=\left(\begin{array}{c}
u(n) \\
w(n)
\end{array}\right)
$$

we rearrange (1) - (3) as the following state-space equations:

$$
\begin{aligned}
\mathbf{x}(n+1) & =\mathbf{F}(n) \mathbf{x}(n)+\mathbf{G} \mathbf{w}(n) \\
y(n) & =\mathbf{c}^{T} \mathbf{x}(n)
\end{aligned}
$$

with $\mathbf{F}(n)=\left(\begin{array}{cc}\mathbf{A}(n) & \mathbf{0} \\ \mathbf{0} & \mathbf{B}(n)\end{array}\right), \mathbf{G}=\left(\begin{array}{cc}\mathbf{e}_{s} & 0 \\ 0 & \mathbf{e}_{v}\end{array}\right), \mathbf{c}=\left(\begin{array}{l}\mathbf{e}_{s} \\ \mathbf{e}_{v}\end{array}\right)$ where unity vector $\mathbf{e}_{s}=(1,0, \ldots, 0)^{T}$ has dimension $d+1$, and $\mathbf{e}_{v}$ has dimension $q$, respectively.

Using the state-space signal model (9), we now design a Kalman filter (smoother) which optimally suppresses the disturbing noise $v(n)$ provided that the model parameter set $\left\{\mathbf{a}(n), \mathbf{b}(n), \sigma_{u}^{2}(n), \sigma_{w}^{2}(n)\right\}$ is known.

The Kalman filter order as given by the $(d+1+q) \times(d+$ $1+q)$ state matrix $\mathbf{F}$ may be reduced by a suitable transformation of the state-vector or of the observation signal $y(n)$ [2]. Unfortunately, the reduced order Kalman filter needs a more complicated Kalman gain vector update. Thus, we decided to stick to the increased order Kalman filter. In most practical situations, the order increase is small because the order $q$ of the disturbing noise model is much less than the smoother delay $d$.

According to (9) the Kalman filter equations for estimation of the state vector $\mathbf{x}(n)$ in (8) may be formulated as

$$
\begin{aligned}
e(n) & =y(n)-\mathbf{c}^{T} \mathbf{F}(n) \hat{\mathbf{x}}(n) \\
\hat{\mathbf{x}}(n+1) & =\mathbf{F}(n) \hat{\mathbf{x}}(n)+\mathbf{k}(n) e(n) \\
\hat{s}(n-d) & =\hat{x}_{d+1}(n) .
\end{aligned}
$$

Vectors $\hat{\mathbf{s}}(n)$ and $\hat{\mathbf{v}}(n)$ are estimates of the subvectors given in (8). The enhanced signal sample $\hat{s}(n-d)$ is obtained by the state vector component with index $d+1$.

The computation of the Kalman gain vector $\mathbf{k}(n)$ can be simplified due to the special structure of $\mathbf{F}(n), \mathbf{G}, \mathbf{c}$. In the following equations, we use a notation for submatrices which can be easily converted to MATLAB syntax:

Let $\mathbf{P}=\mathbf{P}_{(1: d, 1: d)}$ be a $d \times d$ matrix. We will use

$\mathbf{P}_{(1: p, 1: q)} \quad$ to denote the $p \times q$ submatrix containing the first $p$ rows and first $q$ columns of $\mathbf{P}$,

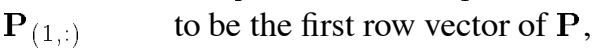

$\mathbf{P}_{(:, 1)} \quad$ to be the first column vector of $\mathbf{P}$,

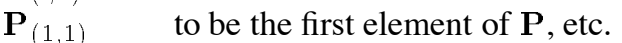

A similar notational convention will be used for subvectors and single vector components. Splitting the Kalman gain vector $\mathbf{k}(n)=\left(\begin{array}{l}\mathbf{k}_{s}(n) \\ \mathbf{k}_{v}(n)\end{array}\right)$ and introducing

$$
\tilde{\mathbf{s}}(n)=\left(\begin{array}{c}
\mathbf{a}^{T}(n) \hat{\mathbf{s}}_{(1: p)}(n) \\
\hat{\mathbf{s}}_{(1: d)}(n)
\end{array}\right), \quad \tilde{\mathbf{v}}(n)=\left(\begin{array}{c}
\mathbf{b}^{T}(n) \hat{\mathbf{v}}(n) \\
\hat{\mathbf{v}}_{(1: q-1)}(n)
\end{array}\right)
$$


yields from (10)

$$
\begin{aligned}
e(n) & =y(n)-\tilde{\mathbf{s}}_{(1)}(n)-\tilde{\mathbf{v}}_{(1)}(n) \\
\hat{\mathbf{s}}(n+1) & =\tilde{\mathbf{s}}(n)+\mathbf{k}_{s}(n) e(n) \\
\hat{\mathbf{v}}(n+1) & =\tilde{\mathbf{v}}(n)+\mathbf{k}_{u}(n) e(n) \\
\hat{s}(n-d) & =\hat{\mathbf{s}}_{(d+1)}(n) .
\end{aligned}
$$

The standard Kalman equations as given e.g. in [2] can be brought into a computationally efficient form by using the above notational convention and a block form of the $(d+1+$ $q) \times(d+1+q)$ error covariance matrix $\mathbf{P}(n)=\left(\begin{array}{cc}\mathbf{S}(n) & \mathbf{X}(n) \\ \mathbf{X}^{T}(n) & \mathbf{V}(n)\end{array}\right)$ :

$$
\begin{aligned}
& \mathbf{p}^{T}(n)=\mathbf{a}^{T}(n) \mathbf{S}_{(1: p, 1: d)}(n) \\
& \widetilde{\mathbf{S}}(n)=\left(\begin{array}{cc}
\mathbf{p}^{T}{ }_{(1: p)}(n) \mathbf{a}(n)+\hat{\sigma}_{u}^{2}(n) & \mathbf{p}^{T}(n) \\
\mathbf{p}(n) & \mathbf{S}_{(1: d, 1: d)}(n)
\end{array}\right) \\
& \mathbf{q}^{T}(n)=\mathbf{b}^{T}(n) \mathbf{V}(n) \\
& \tilde{\mathbf{V}}(n)=\left(\begin{array}{cc}
\mathbf{q}^{T}(n) \mathbf{b}(n)+\hat{\sigma}_{w}^{2}(n) & \mathbf{q}^{T}(1: q-1) \\
\mathbf{q}_{(1: q-1)}(n) & \mathbf{V}_{(1: q-1,1: q-1)}(n)
\end{array}\right) \\
& \mathbf{t}^{T}(n)=\mathbf{a}^{T}(n) \mathbf{X}_{(1: p,:)}(n) \\
& \tilde{\mathbf{X}}(n)=\left(\begin{array}{cc}
\mathbf{t}^{T}(n) \mathbf{b}(n) & \mathbf{t}^{T}(1: q-1) \\
\mathbf{X}_{(1: d,:)}(n) \mathbf{b}(n) & \mathbf{X}_{(1: d, 1: q-1)}(n)
\end{array}\right) \\
& \mathbf{f}(n)=\widetilde{\mathbf{S}}_{(:, 1)}(n)+\tilde{\mathbf{X}}_{(:, 1)}(n) \\
& \mathbf{g}(n)=\tilde{\mathbf{X}}_{(:, 1)}^{T}(n)+\tilde{\mathbf{V}}_{(:, 1)}(n) \\
& \alpha(n)=\frac{1}{\mathbf{f}_{(1)}(n)+\mathbf{g}_{(1)}(n)} \\
& \mathbf{k}_{s}(n)=\alpha(n) \mathbf{f}(n) \\
& \mathbf{k}_{v}(n)=\alpha(n) \mathbf{g}(n) \\
& \mathbf{S}(n+1)=\widetilde{\mathbf{S}}(n)-\mathbf{k}_{s}(n) \mathbf{f}^{T}(n) \\
& \mathbf{V}(n+1)=\tilde{\mathbf{V}}(n)-\mathbf{k}_{v}(n) \mathbf{g}^{T}(n) \\
& \mathbf{X}(n+1)=\tilde{\mathbf{X}}(n)-\mathbf{k}_{s}(n) \mathbf{g}^{T}(n) .
\end{aligned}
$$

The Kalman filter variables are initialized by

$$
\begin{aligned}
\mathbf{S}_{(1,1)}(0) & =\hat{\sigma}_{u}^{2}(0) & \mathbf{S}_{(2: d+1,2: d+1)}(0) & =\mathbf{0} \\
\mathbf{V}_{(1,1)}(0) & =\hat{\sigma}_{w}^{2}(0) & \mathbf{V}_{(2: q, 2: q)}(0) & =\mathbf{0} \\
\mathbf{X}(0) & =\mathbf{0} & & \\
\hat{\mathbf{s}}(0) & =\mathbf{0} & \hat{\mathbf{v}}(0) & =\mathbf{0} .
\end{aligned}
$$

Compared to the white noise case where $\mathbf{P}(n)=\mathbf{S}(n)$ and $\alpha(n)=1 /\left(\mathbf{f}_{(1)}(n)+\hat{\sigma}_{w}^{2}(n)\right)$ the colored noise case requires a lot more computational load. Therefore, we usually restrict the noise AR model order $q$ to small values.

\section{REMOVAL OF IMPULSIVE NOISE}

We now modify the Kalman filter equations to handle signal observations which are corrupted by Gaussian (colored) noise and impulsive noise. The idea of the impulse suppression algorithm is to treat impulses as outliers and to suspend the Kalman filter update during presence of outliers. The impulsive noise will be characterized by short spikes at random time instants. The explicit impulse waveform is not essential for the Kalman filtering operation, since the Kalman filter update is frozen during presence of impulses. However, the impulse detection scheme needed to discriminate Gaussian noise and impulse noise is sensitive in regard to the impulse waveform and amplitude distribution. The impulse detection scheme we adopted for our system is a slightly modified version of a method proposed in [7]. The advantages of this method are its low computational complexity and the fact that only a minor modification of the Kalman filter equations is needed.

The impulse detector is driven by the error signal $e(n)$ as given in (12). Outliers contained in this signal are clearly visible even at moderate additional noise levels. The main problem in the design of the impulse detector is the selection of a threshold level which must be adjusted to signal and noise levels. The threshold level $\theta(n)$ is computed according to the following rule [7]:

$$
\begin{aligned}
\theta(n) & =\mu \frac{E_{e}(n)}{\kappa(n)} \quad \text { with } \\
E_{e}(n) & = \begin{cases}\lambda E_{e}(n-1)+e^{2}(n) & \text { if } e^{2}(n)<\theta(n-1) \\
E_{e}(n-1) & \text { if } e^{2}(n) \geq \theta(n-1)\end{cases} \\
\kappa(n) & = \begin{cases}\lambda \kappa(n-1)+1 & \text { if } e^{2}(n)<\theta(n-1) \\
\kappa(n-1) & \text { if } e^{2}(n) \geq \theta(n-1)\end{cases}
\end{aligned}
$$

with $E_{e}(0)=1$ and $\kappa(0)=1$. Note that an impulse is detected, if $e^{2}(n) \geq \theta(n-1)$ and in that case the threshold value $\theta(n)$ is not updated. The forgetting factor $\lambda$ is usually close to 1 and enables an exponentially decaying data window for energy measurement. Experiments indicate that the threshold factor $\mu$ should be chosen in the range $\mu=9 \ldots 25$.

The only modification applied to the Kalman equations (13) is to freeze the error covariance matrix update and to eliminate the error term in the state vector update (12). We accomplish this by setting gain vectors $\mathbf{k}_{s}(n)$ and $\mathbf{k}_{v}(n)$ to zero vectors during presence of impulses. As a consequence, the Kalman filter operates in an autonomous mode, this is $\hat{\mathbf{x}}(n+1)=$ $\mathbf{F}(n) \hat{\mathbf{x}}(n)$, and discards the outliers by ignoring $e(n)$ in (12) during these time intervals.

\section{ADAPTIVE KALMAN FILTER AND AR PARAMETER ESTIMATION}

Up to now we have assumed that the AR model parameters are known a priori. In practical applications, however, these parameters must be estimated from noisy observations. Therefore, the key point for the performance of our signal enhancement system is the estimation of AR parameters in the presence of noise. In this context, we have studied two different 
techniques to enhance noisy AR signals, and to obtain their AR parameters, respectively. The first technique employs an extended Kalman filter and deals with white noise and impulsive noise disturbances [7]. The extended Kalman filter works well at moderate noise levels. However, for SNRs less than $10 \mathrm{~dB}$ this algorithm exhibits severe convergence problems. A stalling phenomenon may be observed resulting in intermittent signal segments and in slow convergence (as compared to the convergence speed at higher SNRs).

The second approach is based on an iterated parameter estimation and is suited for colored noise disturbances [6]. It is an extension of an algorithm described in [1]. No suppression of impulsive noise has been included in the proposed algorithms. The AR parameters are estimated using an iterative procedure, i.e. filtering of a signal block and parameter estimation are alternated several times. Obviously, this approach results in a high computational complexity. Additionally, in many cases the system performance is getting worse after a certain number of iterations.

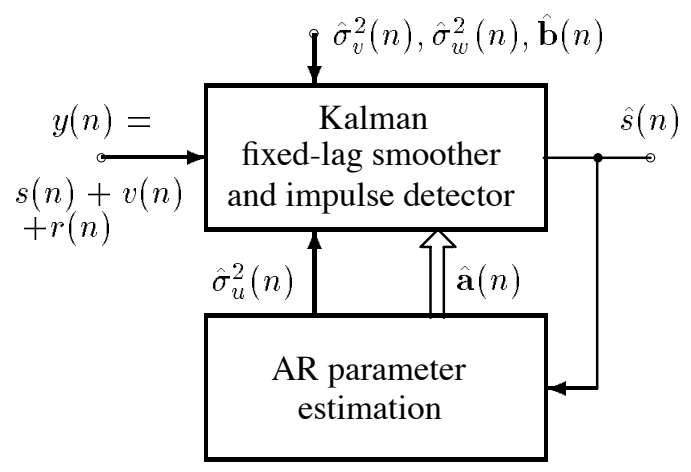

Figure 1: Block diagram of the adaptive Kalman filter for the enhancement of AR signal $s(n)$ disturbed by colored noise $v(n)$ and impulsive noise $r(n)$.

The adaptive Kalman filter used in our enhancement system offers a different approach and has been introduced in [8]. It is based on the adaptive setup as shown in Fig. 1 and may be designed to suppress combinations of colored noise and impulsive noise. The enhanced signal at the output of the Kalman filter is fed to the AR parameter estimation subsystem. At the beginning of the system's convergence period the noisy signal is used for AR parameter estimation. However, after convergence the parameter estimation is carried out on the denoised signal. Convergence of our system is ensured as long as the AR signal contains signal components which are stronger than the disturbing noise. Since the Kalman filter operates as an optimum filter, it emphasizes strong signal components. Due to the feedback loop the parameter estimation of the AR signal is further improved.

We have selected two methods for the AR parameter estimation subsystem in Fig. 1. Method I is a block processing technique where the block length and overlapping is chosen in accordance to the nonstationarity of the AR signal. The Levinson algorithm is applied to overlapping signal blocks for AR parameter estimation [5]. On the contrary, method II employs a recursive least-squares lattice (LSL) algorithm [4]. The LSL algorithm operates on a sample per sample basis and in case of an exponential error weighting the time constant (forgetting factor) determines the tracking behavior of the algorithm. With the Levinson algorithm, stability of the AR model is guaranteed. In general, the recursive LSL algorithm with exponential error weighting may produce unstable AR filters. However, stability can be easily checked by observing the reflection coefficients of the LSL algorithm. In addition, in all our experiments with a wide range of AR signals we have never encountered any stability problems.

The estimation of $\sigma_{u}^{2}$ is a crucial point since its value determines the noise reduction behavior and the convergence speed of the adaptive Kalman filter. Furthermore, we must use a lower bound $\sigma_{\min }^{2} \neq 0$ for $\hat{\sigma}_{u}^{2}$ since $\hat{\sigma}_{u}^{2}=0$ prevents a proper start up of the algorithm (see (13) and (14)). If $\sigma_{\min }^{2}$ is chosen too small, then the signal enhancement is certainly better but convergence is slow in general, and vice versa.

A performance analysis of the adaptive Kalman filter shown in Fig. 1 has been carried out in [8] in some detail. The influence of white noise on the AR parameter estimation has been found to be the major performance limiting factor. A similar result holds for colored input noise, since the whitening property of the Kalman filter produces a white output noise. Compared to the white input noise case investigated in [8], however, suppression of colored input noise requires a longer settling time of the Kalman filter and results in less SNR improvements. These effects are supported by experimental results and are more pronounced in case of spectral similarities between desired signal and colored noise.

\section{EXPERIMENTAL RESULTS}

We illustrate the performance of our enhancement system by two typical experimental results. The first example is shown in Fig. 2. The clean signal is composed of a $p=4$ order AR signal (time interval $n=0 \ldots 999$ ) and a $p=3$ order AR signal (time interval $n=1000 \ldots$. 1999). A fixed AR model order $p=8$ is used by the enhancement system. The measurement of the colored noise parameters $\sigma_{w}^{2}(n), \mathbf{b}(n)$ is carried out before the desired (clean signal in Fig. 2 (a)) is switched on. The disturbing noise is an AR process of order $q=1$ plus an impulsive noise with constant amplitude $A=1$. The parameters of the automatic impulse detector are set to $\lambda=0.99$ and $\mu=12.25$. The second example (Fig. 3) shows the suppression of a strong low frequency interference due to a colored noise with $q=1$ and a pole location $p_{\infty}=0.99$. The desired signal consists of a $p=4$ order AR signal switched to a $p=2$ order AR signal at $n=500$. In both examples, the tracking capability of the adaptive Kalman filter is clearly visible (signal traces Fig. 2 
(c) and Fig. 3 (c)). The computational complexity of example I simulated with MATLAB is 4058 FLOPS per sampling period, and 3711 FLOPS for example II, respectively.

\section{CONCLUSIONS}

A computationally efficient adaptive Kalman smoother for enhancing AR signals corrupted by colored noise and impulses has been presented. The system was originally designed for white noise disturbances only. As shown in this paper, however, it can be rather easily extended to cover more realistic types of noises. The system requires a minimum a priori knowledge of signal and noise parameters and is capable of tracking short-time stationary signals.

\section{REFERENCES}

[1] J. S. Lim, A. V. Oppenheim, "All-Pole Modeling of Degraded Speech", IEEE Trans., Vol. ASSP-26, June 1978, pp. 197-210.

[2] B. D. O. Anderson, J. B. Moore, "Optimal filtering", Chapters 7, 11, Prentice-Hall, Inc., 1979.

[3] G. C. Goodwin, "Adaptive filtering prediction and control", Chapter 7, Prentice-Hall, Inc., 1984.

[4] S. Haykin, "Adaptive filter theory", Chapter 9, Prentice-Hall, Inc., 1987.

[5] S. L. Marple Jr., "Digital Spectral analysis with applications", Chapter 7, Prentice-Hall, Inc., 1987.

[6] J. D. Gibson, B. Koo, S. D. Gray, "Filtering of Colored Noise for Speech Enhancement and Coding", IEEE Trans. on Signal Processing, Vol. 39, Aug. 1991, pp. 1732-1742.

[7] M. Niedźwiecki, K. Cisowski, "Adaptive scheme for elimination of broadband noise and impulsive disturbances from AR and ARMA signals", IEEE Trans. on Signal Processing, Vol. 44, March 1996, pp. 528-537.

[8] G. Doblinger, "An adaptive Kalman filter for the enhancement of noisy AR signals", Proc. 1998 IEEE Int. Symp. ISCAS '98, May 31-June 3, 1998.
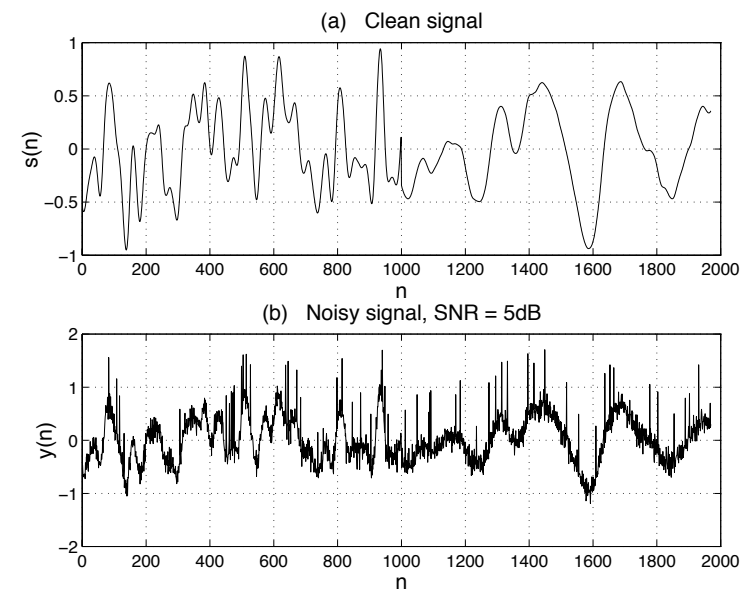

(c) Enhanced signal

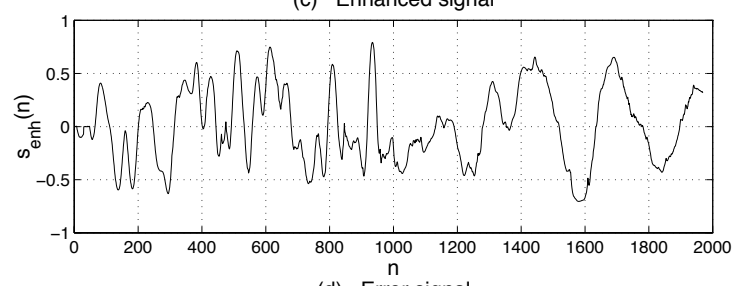

(d) Error signal

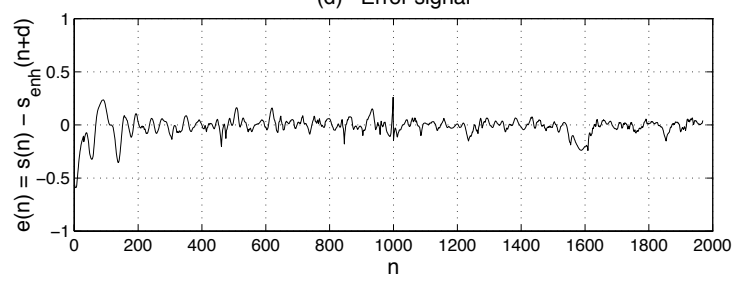

Figure 2: Example I: Colored noise plus impulsive noise disturbances (adaptive Kalman filter with $p=8$, Levinson algorithm for AR parameter estimation, smoother delay $d=30$, block length $N=300$, AR parameters computed every 10 samples).

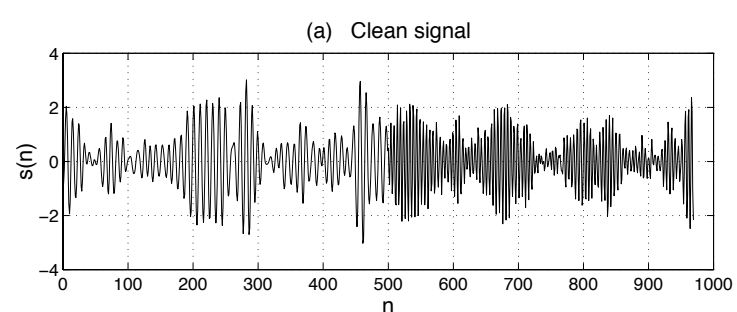

(b) Noisy signal, $\mathrm{SNR}=5 \mathrm{~dB}$

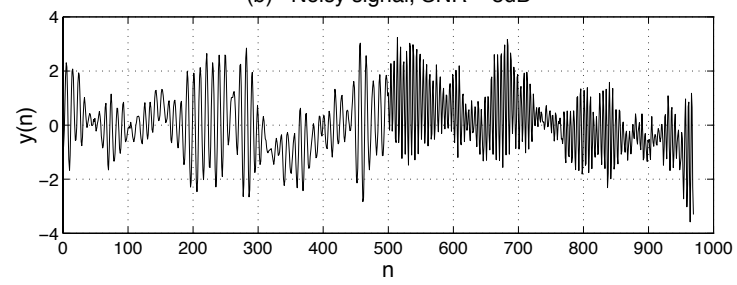

(c) Enhanced signal

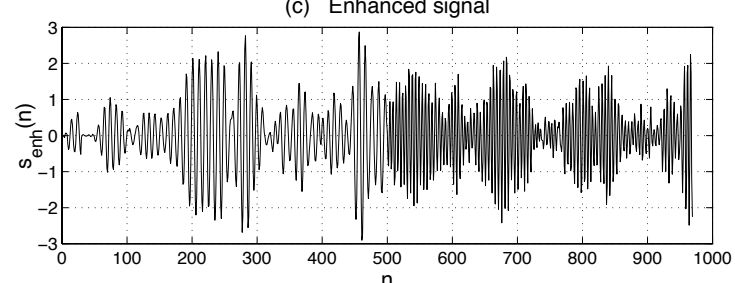

(d) Estimation error signal $\mathrm{e}(\mathrm{n})$ and disturbing noise $\mathrm{v}(\mathrm{n})$

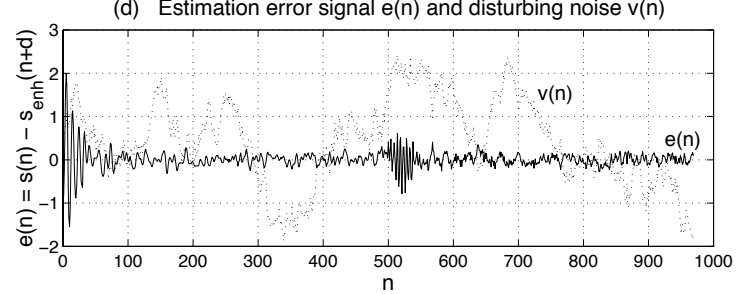

Figure 3: Example II: Colored noise disturbance (adaptive Kalman filter with $p=4$, remaining parameters as in example I). 